\title{
High-performance $\mathrm{Ba}_{1-x} \mathrm{~K}_{x} \mathrm{Fe}_{2} \mathrm{As}_{2}$ superconducting tapes with grain texture engineered via a scalable fabrication
}

\author{
Shifa Liu ${ }^{1,2}$, Chao Yao ${ }^{1,2^{*}}$, He Huang ${ }^{1}$, Chiheng Dong ${ }^{1,2}$, Wenwen Guo ${ }^{1,2}$, Zhe Cheng ${ }^{1,2}$, \\ Yanchang $\mathrm{Zhu}^{1}$, Satoshi Awaji ${ }^{3}$ and Yanwei $\mathrm{Ma}^{1,2^{*}}$
}

\begin{abstract}
Nowadays the development of high-field magnets strongly relies on the performance of superconducting materials. Iron-based superconductors (IBSs) exhibit high upper critical fields and low electromagnetic anisotropy, making them particularly attractive for high-field applications, especially in particle accelerator magnets, nuclear magnetic resonance spectrometers, medical magnetic resonance imaging systems and nuclear fusion reactors. Herein, through an industrially scalable manufacturing strategy, a practical-level critical current density up to $1.1 \times 10^{5} \mathrm{~A} \mathrm{~cm}^{-2}$ at $4.2 \mathrm{~K}$ in an external magnetic field of $10 \mathrm{~T}$ was achieved in $\mathrm{Cu} /$ Ag composite-sheathed $\mathrm{Ba}_{1-x} \mathrm{~K}_{x} \mathrm{Fe}_{2} \mathrm{As}_{2} \quad$ (Ba122) superconducting tapes. The preparation strategy combines flat rolling to induce grain texture and subsequent hot-isostaticpressing densification. By varying the parameters of rolling, the degree of grain texture was engineered. It is found that the transport properties of the Ba122 tapes can be enhanced by applying a large amount of deformation during rolling, which can be attributed to the improved degree of $c$-axis texture. Microstructure characterizations on the highest-performance tape demonstrate that the Ba122 phase has a uniform element distribution and small grains with good connectivity. Grain boundary pinning is consequently enhanced as proved by large currents circulating through the sample even at $25 \mathrm{~K}$. Our work proves that $\mathrm{Cu} / \mathrm{Ag}$ composite-sheathed Ba122 superconducting tapes can be a promising competitor for practical high-field applications in terms of the viable, scalable and cost-effective fabrication strategy applied and the high transport properties achieved in this work.
\end{abstract}

Keywords: iron-based superconductors, critical current density, scalable, grain texture, hot isostatic pressing

\section{INTRODUCTION}

Iron-based superconductors (IBSs) discovered in 2008 have attracted much attention due to their unique superconducting mechanism and potential value in practical applications [1-3]. To date, among several tens of reported IBSs [4-6], "122" iron-pnictide compounds such as $\mathrm{Sr}_{1-x} \mathrm{~K}_{x} \mathrm{Fe}_{2} \mathrm{As}_{2}$ (Sr122) and $\mathrm{Ba}_{1-x} \mathrm{~K}_{x} \mathrm{Fe}_{2} \mathrm{As}_{2}$ (Ba122) hold great promise for high-field magnet applications $[7,8]$, because they have moderately high critical transition temperatures $T_{\mathrm{c}}$ (up to $38 \mathrm{~K}$ ), high upper critical fields $H_{\mathrm{c} 2}$ (above $75 \mathrm{~T}$ ) and low anisotropy $\gamma(<2)$ [8-11]. For such applications, superconducting wires and tapes with high in-field transport critical current densities $\left(J_{c}\right)$ are crucial. Tremendous advances have been made in $J_{c}$ enhancement of iron-pnictide wires and tapes prepared by a powder-in-tube (PIT) method over the past decade $[4,12,13]$. In order to obtain high $J_{\mathcal{c}}$, besides high-quality precursor powder, there are two major issues that need to be resolved, namely the poor connectivity of Ba122 grains due to the microstructural defects such as pores and cracks which are extrinsically induced during the PIT process and intrinsic weak links at the high-angle grain boundaries between mismatched grains [14,15]. Pores and cracks hinder the supercurrent flow inside superconducting polycrystals, while weak-link behavior results in an exponential decay of inter-grain $J_{\mathrm{c}}$ as a function of grain boundary angle when it is larger than a so-called critical angle $\left(\theta_{c}\right)[16]$. Actually, Ba122 exhibits a much higher tolerance for mismatched grains than $R E \mathrm{Ba}_{2} \mathrm{Cu}_{3} \mathrm{O}_{7}$ $(R E=$ rare earth elements) cuprate superconductors [1719]. The $\theta_{c}$ for cobalt-doped $\mathrm{BaFe}_{2} \mathrm{As}_{2}$ epitaxial films is about $9^{\circ}$, larger than the $\theta_{c}$ of $5^{\circ}$ for $\mathrm{YBa}_{2} \mathrm{Cu}_{3} \mathrm{O}_{7-\delta}$. Even

\footnotetext{
${ }^{1}$ Key Laboratory of Applied Superconductivity, Institute of Electrical Engineering, Chinese Academy of Sciences, Beijing 100190, China

${ }^{2}$ University of Chinese Academy of Sciences, Beijing 100049, China

${ }^{3}$ High Field Laboratory for Superconducting Materials, Institute for Materials Research, Tohoku University, Sendai 980-8577, Japan

* Corresponding authors (emails: yaochao@mail.iee.ac.cn (Yao C); ywma@mail.iee.ac.cn (Ma Y))
} 
for misorientation angles larger than $\theta_{c}$, cobalt-doped Ba122 films show a much slower decay of $J_{\mathrm{c}}$ than $\mathrm{YBa}_{2}$ $\mathrm{Cu}_{3} \mathrm{O}_{7-\delta}$. On the other hand, measurements on Ba122 bulks reveal that a large $J_{c}$ results from small grains [20]. This attribute even has called into question whether grain texture is still crucial or not for high $J_{c}$-performance in iron-pnictide wires and tapes.

In " 122 " iron-pnictide tapes, the $c$-axis texture can be mechanically introduced by deforming round wires into thin tapes through flat rolling [21,22]. In order to explicitly clarify the role of grain texture in $J_{c}$ improvement for Ba122 tapes, some extrinsic ingredients such as impurity phases, composition inhomogeneity, pores and cracks should be eliminated as much as possible. With the uniaxial pressing densification process, a high transport $J_{\mathrm{c}}$ above $10^{5} \mathrm{~A} \mathrm{~cm}^{-2}$, which is a widely accepted threshold for practical applications, has been reported in $c$-axistextured Sr122 and Ba122 iron-pnictide tape samples [2325]. However, strictly limited by the size of pressing heads, this manufacturing route is problematic to be scaled up for processing long wires and tapes. Moreover, the widely used uniaxial pressing, either cold pressing or hot pressing, not only enhances the mass density, but also further improves the grain texture simultaneously, making it complicated to investigate the contribution of mass densification and grain alignment to the $J_{c}$-performance separately.

As an alternative to uniaxial pressing for densification, the hot isostatic pressing (HIP) process utilizing highpressure argon gas as media can effectively reduce the pores and cracks in superconducting cores. The $J_{c}$ values of $\sim 10^{4} \mathrm{~A} \mathrm{~cm}^{-2}$ at $4.2 \mathrm{~K}$ and $10 \mathrm{~T}$ were measured in HIP round wires with randomly oriented grains [26,27]. The mass density of iron-pnictide cores of wires and tapes can be indicated with Vickers hardness. It is reported that the mass density of Ba122 cores in HIP processed wires can be significantly enhanced to nearly $100 \%$, showing Vickers hardness even higher than that in uniaxially pressed tapes [24,26-29]. Compared with uniaxial pressing, HIP technique is much more flexible and economic for large-scale producing, since long wires and tapes can be processed in winding state in space-limited furnaces. However, it can hardly induce any grain alignment as done by rolling and uniaxial pressing, and further efforts over the past several years have failed to improve $J_{c}$ values of HIP-treated round wires to a practical level at a field of $10 \mathrm{~T}$.

For Ba122 superconducting tapes, grain alignment introduced by the rolling process can be well preserved after heat treatment by the HIP densification process [30].
Herein, the flat rolling and a subsequent HIP process were combined to obtain $c$-axis texture and well-connected superconducting grains. Since mass densification and $c$-axis texture were achieved through two separate processes, the effect of grain alignment on transport $J_{c}$ can be studied based on highly dense Ba122 phase. The starting wire diameters for rolling were varied to induce different degrees of texture for the purpose of revealing the correlation between grain textures and transport $J_{\mathrm{c}}$ in Ba122 tapes. Besides, copper was used as the sheath material to partically replace silver in purpose of reducing the fabrication cost and enhancing the mechanical strength of the tapes. Finally, as a result, $J_{c}$ was enhanced to $1.1 \times 10^{5} \mathrm{~A} \mathrm{~cm}^{-2}$ at $4.2 \mathrm{~K}$ and $10 \mathrm{~T}$, which confirms the indispensability of texture for high performance in Ba122 tapes.

\section{EXPERIMENTAL SECTION}

Ba122 tapes were prepared by the ex situ PIT method. The preparation of $\mathrm{Ba}_{1-x} \mathrm{~K}_{x} \mathrm{Fe}_{2} \mathrm{As}_{2}(x=0.4)$ precursor was detailed in the Ref. [30]. Fig. 1a-c illustrate the PIT and cold working deformation process. The precursor was ground into powder using an agate mortar, and sealed into a silver tube with an outer diameter of $8.0 \mathrm{~mm}$ and an inner diameter of $5.0 \mathrm{~mm}$. These steps were done in an argon-filled glovebox to avoid contact with oxygen and moisture. The silver tube was first swaged into an outer diameter of $3.4 \mathrm{~mm}$ and then drawn into a wire with an outer diameter of $1.93 \mathrm{~mm}$. The wire was cut into several pieces and each piece was sealed into a copper tube with an outer diameter of $4.0 \mathrm{~mm}$ and an inner diameter of $2.0 \mathrm{~mm}$. The $\mathrm{Cu} / \mathrm{Ag}$ composite-sheathed wires were again deformed by swaging and drawing. The final diameters of these wires were $1.3,1.5,1.7$ and $1.9 \mathrm{~mm}$ respectively. After that, these wires were rolled into tapes with thickness of $0.3 \mathrm{~mm}$ after 4 or 5 passes. Finally, these tapes were sintered for $1 \mathrm{~h}$ at $740^{\circ} \mathrm{C}$ by $\mathrm{HIP}$ at $150 \mathrm{MPa}$ in argon atmosphere.

The field-dependent critical current measurements were performed at the High Field Laboratory for Superconducting Materials at Sendai by a standard four-probe method, with a criterion of $1 \mu \mathrm{V} \mathrm{cm}{ }^{-1}$. It needs to be pointed out that the test was carried out under decreasing fields. For further analysis, several short pieces of these Ba122 tapes were chemically treated to remove the metal sheath to obtain the bare Ba122 cores or were embedded in conductive resin and mechanically ground and polished. The Vickers hardness testing was performed on the polished cross sections by using the Wilson 402MVD tester. The magnetic-field- and temperature-dependent 


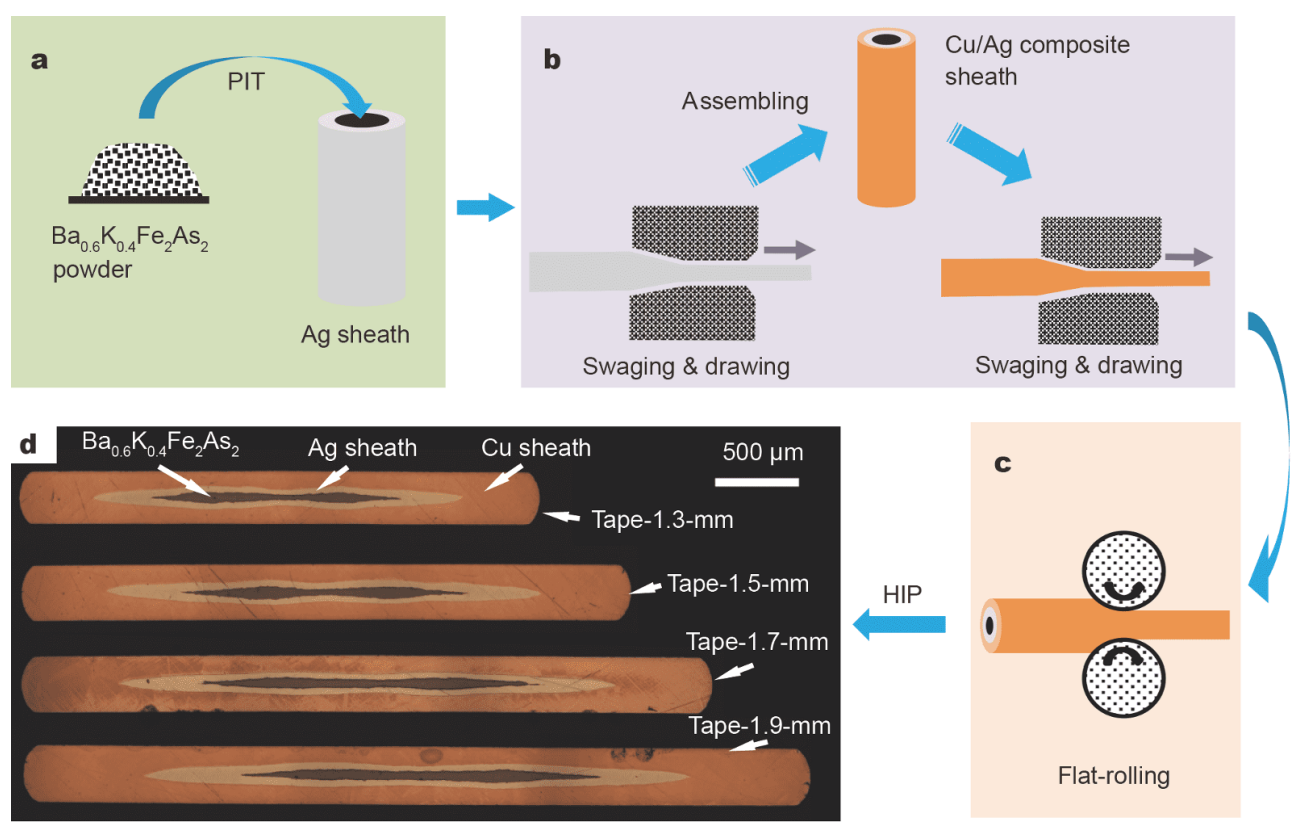

Figure 1 (a-c) Illustrations of the fabrication process of $\mathrm{Cu} / \mathrm{Ag}$ composite-sheathed Ba122 tapes. (d) Optical images of typical cross sections of $\mathrm{Cu} /$ Ag composite-sheathed Ba122 tapes. These tapes were flat rolled from round wires with diameters of 1.3, 1.5, 1.7 and $1.9 \mathrm{~mm}$, respectively.

resistance of the Ba122 cores was measured by a quantum design physical property measurement system (PPMS). The magnetic property of the tape was characterized by SQUID-VSM on the magnetic property measurement system (MPMS). X-ray diffraction (XRD, Bruker D8 Advance) measurements were conducted on the surfaces of the Ba122 cores with $\mathrm{Cu} \mathrm{Ka}{ }_{1}$ radiation. The scanning electron microscopy (SEM, Zeiss SIGMA) observation was performed on the cross sections of the Ba122 cores after they were mechanically broken. By using an EDAX Hikari camera equipped on the SEM, the grain texture was quantitatively represented by the electron backscatter diffraction (EBSD) technique. Vibratory polishing was applied on the sample for EBSD analysis to eliminate the stress of the polished surface. The element distribution on the polished cross section was analyzed by an electron probe micro-analyzer (EPMA, JXA-iSP100). The magneto-optical $(\mathrm{MO})$ imaging was performed on a cryostat (Montana Instruments) by placing a Bi-substituted irongarnet film on the top of the polished superconducting core. Magnetic fields perpendicular to the sample surface were provided by a homemade magnet.

\section{RESULTS}

Fig. 1d shows the optical images of the four Ba122 superconducting tapes rolled from round wires with different diameters of $1.3,1.5,1.7$ and $1.9 \mathrm{~mm}$. The asobtained tapes are labeled as tape-1.3- $\mathrm{mm}$, tape-1.5- $\mathrm{mm}$, tape-1.7-mm and tape-1.9-mm, respectively. The typical cross-sectional images present a Ba122 superconducting core surrounded by an inner silver barrier and an outer copper sheath. In order to quantify the amount of deformation during rolling, a reduction ratio $r$ can be defined as $r=\left(D_{0}-d\right) / D_{0}$, where $D_{0}$ is the initial diameter and $d$ is the final tape thickness (in this work $d=$ $0.3 \mathrm{~mm}$ ). The calculated $r$ values for tape-1.3-mm, tape$1.5-\mathrm{mm}$, tape-1.7-mm and tape-1.9-mm are 0.77, 0.80, 0.82 and 0.84 , respectively. Vickers hardness of the Ba122 cores was measured on the cross sections of the tapes. The average values for these tapes are 202, 197, 203 and 212 , respectively, which are comparable to that of the previously reported HIP samples [27,30]. The high Vickers hardness usually indicates a high mass density of the superconducting cores.

In Fig. 2a, $J_{\mathrm{c}}$ values of the four Ba122 tapes were plotted as a function of the applied magnetic field. Among the four tapes, transport $J_{c}$ of tape-1.9-mm is the highest, reaching $1.1 \times 10^{5} \mathrm{~A} \mathrm{~cm}^{-2}$ at $4.2 \mathrm{~K}$ and $10 \mathrm{~T}$, which is a record value for $\mathrm{Cu} / \mathrm{Ag}$ composite-sheathed IBS tapes and surpasses the threshold for practical applications. It is still above $10^{5} \mathrm{~A} \mathrm{~cm}^{-2}$ even at $12 \mathrm{~T}$, and slightly decreases to $9.4 \times 10^{4} \mathrm{~A} \mathrm{~cm}^{-2}$ at $14 \mathrm{~T}$, showing very weak field dependence. For tape-1.7-mm, tape-1.5-mm and tape-1.3-mm, the $J_{\mathrm{c}}$ values are $9.5 \times 10^{4}, 9.2 \times 10^{4}$ and $5.8 \times 10^{4} \mathrm{~A} \mathrm{~cm}^{-2}$ at $4.2 \mathrm{~K}$ and $10 \mathrm{~T}$, respectively. It is obvious that when the initial diameter of the as-drawn wires becomes smaller, 

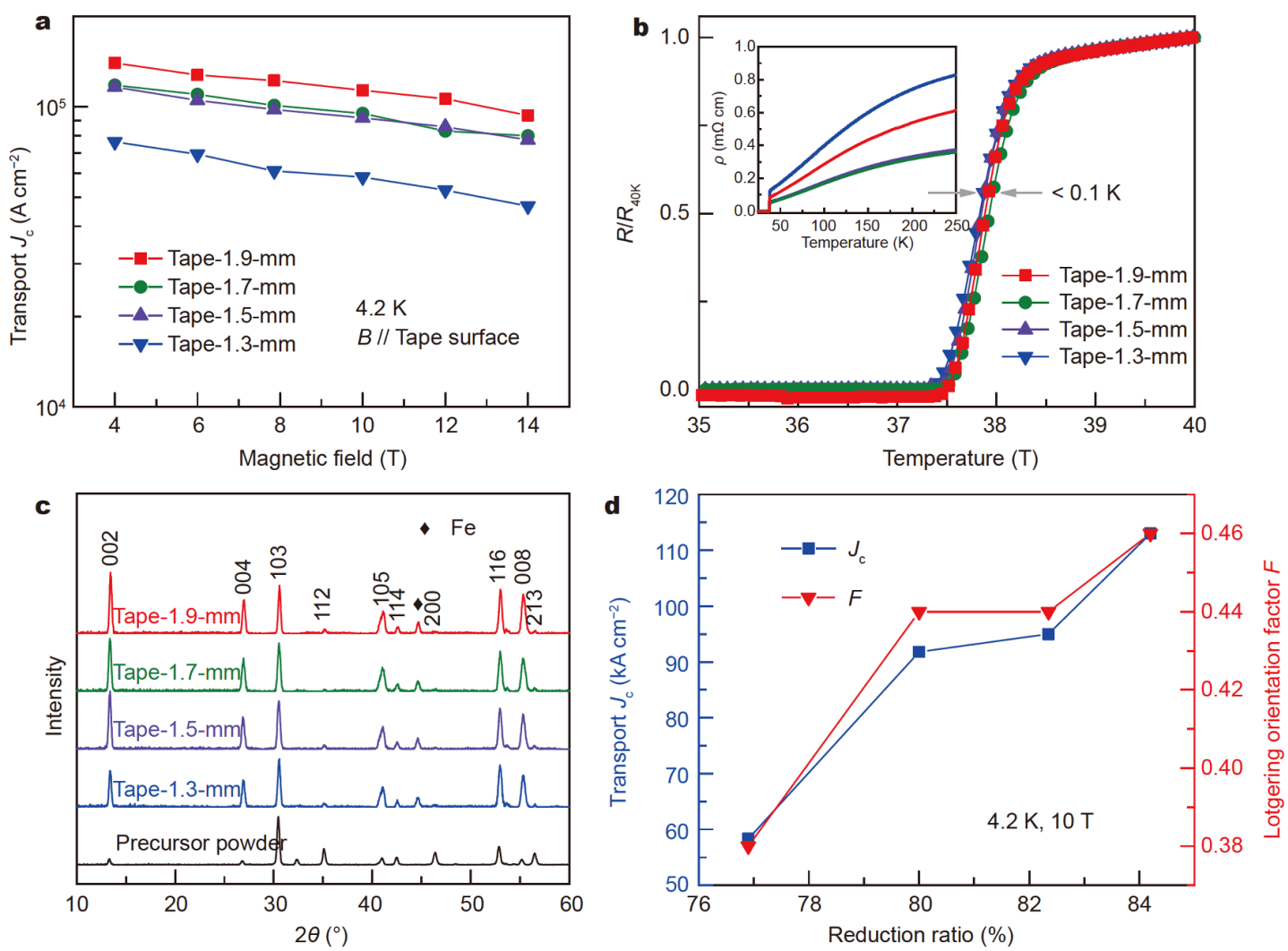

Figure 2 (a) Critical current density as a function of the applied magnetic field for tape-1.9-mm, tape-1.7-mm, tape-1.5-mm, tape-1.3-mm. (b) The temperature-dependent resistivity of the tapes in the self-field. (c) XRD patterns of precursor powder and the cores of the tapes. The $\mathrm{Ba}_{0.6} \mathrm{~K}_{0.4} \mathrm{Fe}_{2} \mathrm{As}_{2}$ diffraction peaks have been indexed. Solid rhombus indicates the diffraction peaks related to iron. (d) Transport $J_{\mathrm{c}}(4.2 \mathrm{~K}, 10 \mathrm{~T})$ and Lotgering orientation factor $F$ as a function of the reduction ratio $r$.

i.e., the reduction ratio is lowered, the $J_{c}$ values of the tapes decrease. Fig. $2 \mathrm{~b}$ shows the resistance transition curves of the four tapes in the self-field. The inset in Fig. $2 \mathrm{~b}$ shows the resistivity of the four tapes as a function of temperature in a range from 25 to $250 \mathrm{~K}$. It is worth noting that the resistance measurements were performed on the bare Ba122 cores, and thus the result reflects the intrinsic property of the Ba122 phase in the tapes. For comparison, the resistivity of each tape is normalized by dividing by its resistivity value at $40 \mathrm{~K}$. A distinctive feature of the resistance transition curves is that the four curves almost overlap with a $T_{\mathrm{c}}$ difference within $0.1 \mathrm{~K}$. Therefore, it can be inferred that the Ba122 polycrystals in these tapes have little difference in the element content and phase purity. The onset $T_{\mathrm{c}}$ values of the four tapes are all around $38.2 \mathrm{~K}$, indicating that high-quality Ba122 phase was obtained in these tapes.

The grain orientation and phase composition for the Ba122 tapes were investigated by XRD, as shown in Fig. 2c. The pattern of the randomly oriented Ba122 precursor powder is also presented for comparison. Generally, for all the samples, Ba122 phase is the main phase, while in the tape samples, diffraction peaks of iron were also detected, which is ascribed to unreacted iron formed in HIP sintering. The intensity of all the XRD patterns was normalized by the intensity of the (103) peak of Ba122 phase. Compared with the precursor powder, the intensity of $(00 l)$ peaks in these tapes is strongly increased, which is significant evidence of the presence of $c$ axis texture in these tapes. According to the Lotgering method [31], the degree of texture can be evaluated by an orientation factor $F=\left(\rho-\rho_{0}\right) /\left(1-\rho_{0}\right)$, where $\rho=\sum I(00 l) /$ $\sum I(h k l), \rho_{0}=\sum I_{0}(00 l) / \sum I_{0}(h k l), I$ and $I_{0}$ are the intensities of each reflection peak $(h k l)$ in the range of $2 \theta$ from $10^{\circ}$ to $60^{\circ}$ for the tape samples and the randomly oriented precursor powder, respectively. For tape-1.3$\mathrm{mm}$, tape-1.5-mm, tape-1.7- $\mathrm{mm}$ and tape-1.9- $\mathrm{mm}$, the calculated $F$ values are $0.38,0.42,0.42$ and 0.46 , respectively. In Fig. $2 \mathrm{~d}$, the transport $J_{\mathrm{c}}(4.2 \mathrm{~K}, 10 \mathrm{~T})$ and Lotgering orientation factor $F$ are plotted as a function of the 
reduction ratio $r$. It clearly shows that the Ba122 tapes with a larger reduction ratio have a higher degree of texture and a higher transport $J_{c}$, and there is a positive and very strong correlation between grain texture and

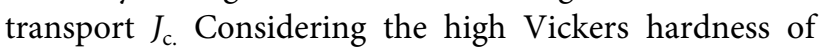
around 200 for the superconducting cores in all the tapes, it can be inferred that high texture plays an important role in the realization of high $J_{c}$-performance.

In order to link the high transport properties in these tapes to their microstructures, detailed characterizations were carried out on the sample tape-1.9-mm, which possesses the highest $J_{c}$ value. First of all, the grain morphology of the tape was viewed on the cross section by SEM, as shown in Fig. 3a-c. Fig. 3b is a partially enlarged view of the area in the white dash-line frame. To describe the spatial relationship between the viewing angle and the tape, three principal axes are defined, the normal direction (ND), the rolling direction (RD) and the transverse direction (TD), as shown in the inset of Fig. $3 \mathrm{~b}$. The images clearly show the influence of rolling process on the grain alignment in the tapes. The maximum thickness of the core is about $60 \mu \mathrm{m}$ with plate-like grains of about $5 \mu \mathrm{m}$ in size. These plate-like grains are aligned along a direction approximately parallel to the tape surface due to the pressure component in the ND applied during the rolling process. Fig. $3 c$ shows that the growth of Ba122 grains is suppressed near the interface between the silver barrier and the Ba122 core as indicated by the white arrow. Further element distribution mapping was performed on the polished cross section of tape-1.9-mm through the EPMA technique, as shown in Fig. $3 d-i$. The Ag mapping shows that near the Ag-Ba122 interface, there is a $1-3 \mu \mathrm{m}$ wide area where Ba122 and Ag coexist, as can be seen in Fig. 3i. The secondary electron image (SEI) in Fig. 3d shows a dense Ba122 phase embedded in the silver layer. A few defects were also observed. The white arrows indicate several small holes and the red oval indicates a barium-riched particle as verified by the distribution map of barium. The particle in the green oval with a diameter of around $3 \mu \mathrm{m}$ comes probably from the polishing suspension. As shown in Fig. 3e-h, except for the barium-riched area, all the four elements $(\mathrm{Ba}, \mathrm{K}, \mathrm{Fe}$ and As) are uniformly distributed, which is crucial for the realization of high transport $J_{c}$.

Despite the XRD and SEM results presenting strong evidence for the $c$-axis texture of the Ba122 grains in these tapes, quantitative and visualized characterizations through EBSD technique performed on the well-polished cross section of tape-1.9- $\mathrm{mm}$ can further provide valuable information. Fig. $4 \mathrm{a}$ shows the inverse pole figure (IPF)
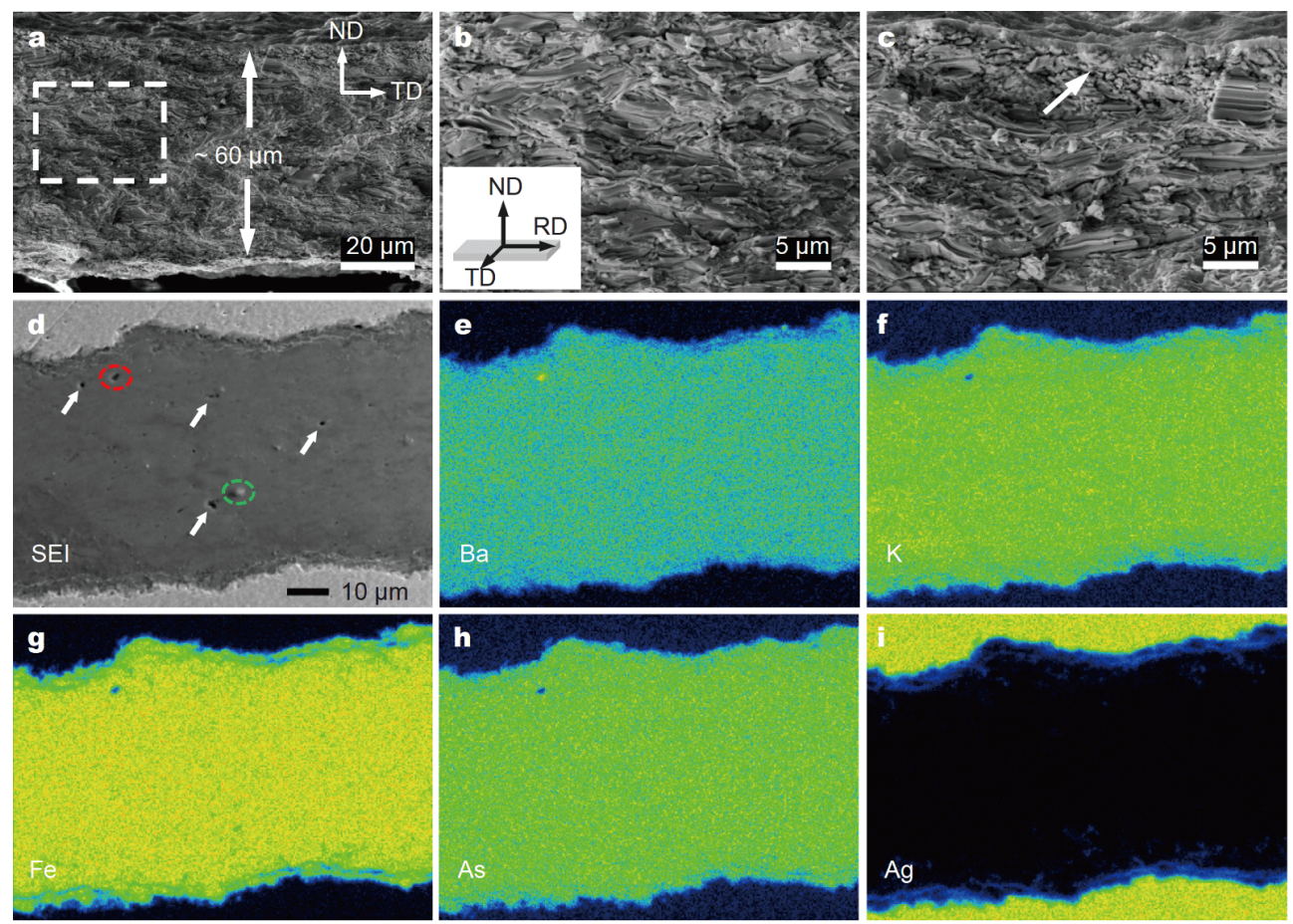

Figure 3 Typical SEM images showing the plate-like grain morphology of (a) the core of tape-1.9-mm, and the partially enlarged view (b) in the center as indicated by the white dash-line frame and (c) near the edge. The inset in (b) shows the three principal axes defined as ND, RD and TD. (d) The secondary electron image and (e-i) element distribution maps on the cross section of tape-1.9-mm. 
$\mathbf{a}$

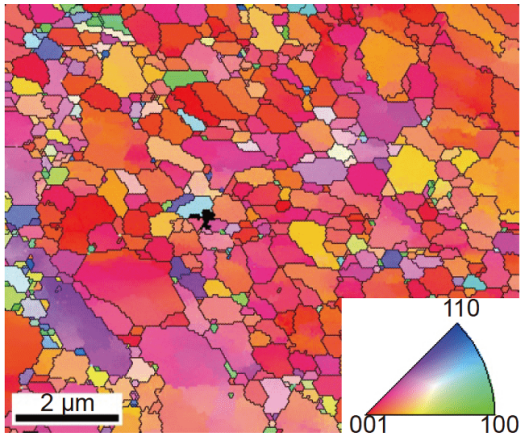

b

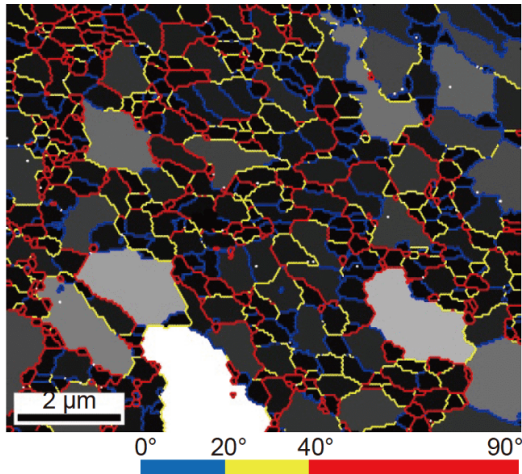

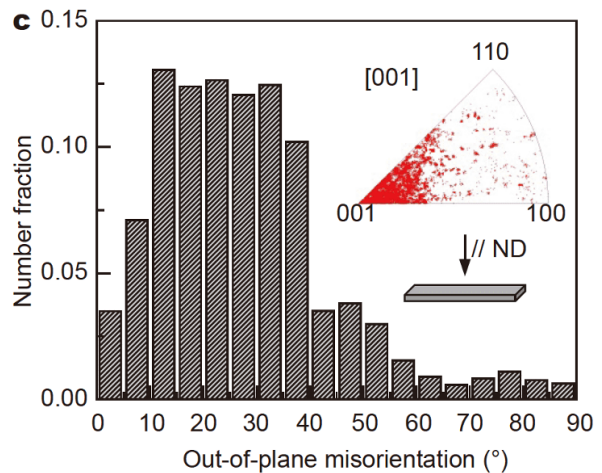

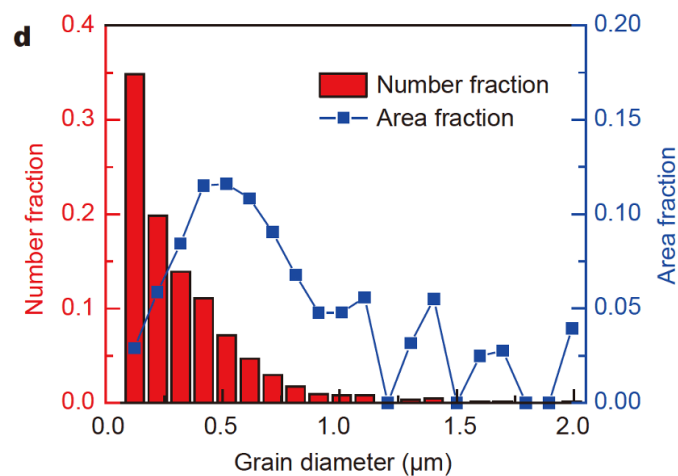

Figure 4 Characterization of the grain structure of tape-1.9-mm viewed from the ND of the tape surfaces. (a) The IPF maps in [001] direction for tape-1.9-mm. (b) The grain size map on gray scales ranging from black to white on increasing grain size. The grain boundaries are colored based on the grain misorientation angles. (c) The distribution of out-of-plane misorientations in the selected area. The inset shows the ND-IPF derived from IPF map in (a). (d) The distribution of the grain size of tape-1.9-mm is presented in two ways: the number fraction and the area fraction.

map in (001) direction of a selected area viewed from the direction parallel to ND. The Ba122 grains are colored according to their grain orientations using a color cord given in the stereographic triangle. The dominant colors of the IPF map are red and reddish, which correspond to $c$-axis texture. Quantitative statistics of the distribution of out-of-plane misorientation angles are shown in Fig. 4c. In this sample, the majority of the out-of-plane misorientation angles are distributed from $5^{\circ}$ to $40^{\circ}$, showing a relatively low degree of texture than in hot-pressed tapes [25]. The inset in Fig. $4 \mathrm{c}$ is the IPF of grain orientations. Fig. $4 \mathrm{~b}$ presents Ba122 grains in the same location as Fig. 4a with gray scales ranging from black to white indicating the grain size. Grain boundaries in Fig. $4 \mathrm{~b}$ are colored based on the grain misorientation angles, as shown in the legend below. The distribution of grain size is given in Fig. 4d in two ways: number fraction and area fraction. The grain size ranges from 0.1 to $2.0 \mu \mathrm{m}$, which is smaller than that in hot-pressed tapes [25]. Grains around $0.5 \mu \mathrm{m}$ in size account for the largest proportion of the total area, and most of them are roughly $c$-axis textured. Besides, there are also a few relatively larger grains, about 1-2 $\mu \mathrm{m}$ in size. However, as Fig. 4a and $b$ show, lots of tiny grains (smaller than $0.2 \mu \mathrm{m}$ in size) are randomly oriented with high-angle grain boundaries, which is definitely an impediment of the transport currents. This can be attributed to the relatively low sintering temperature and short holding time of the HIP process that may not be beneficial to the growth of grains.

MO imaging is a powerful tool that can visualize the distribution of magnetic flux. Through this method, one can directly observe the real-time magnetic field profile produced by the magnetization currents flowing in a superconductor and gain a wealth of information on homogeneity, grain connectivity and the intra- and intergranular $J_{c}$. Fig. 5a shows the optical image of the sample for MO imaging, and its dimension is $1.75 \mathrm{~mm} \times 1.30 \mathrm{~mm} \times 0.025 \mathrm{~mm}$. Fig. $5 \mathrm{~b}-\mathrm{f}$ show the $\mathrm{MO}$ images of the sample at Meissner state acquired by applying an external field on the zero-field cooled sample. At fields less than $125 \mathrm{Oe}\left(1 \mathrm{Oe}=79.577 \mathrm{~A} \mathrm{~m}^{-1}\right)$, the magnetic flux was excluded from the bulk due to the large shielding currents, except for a breakthrough at a crack 

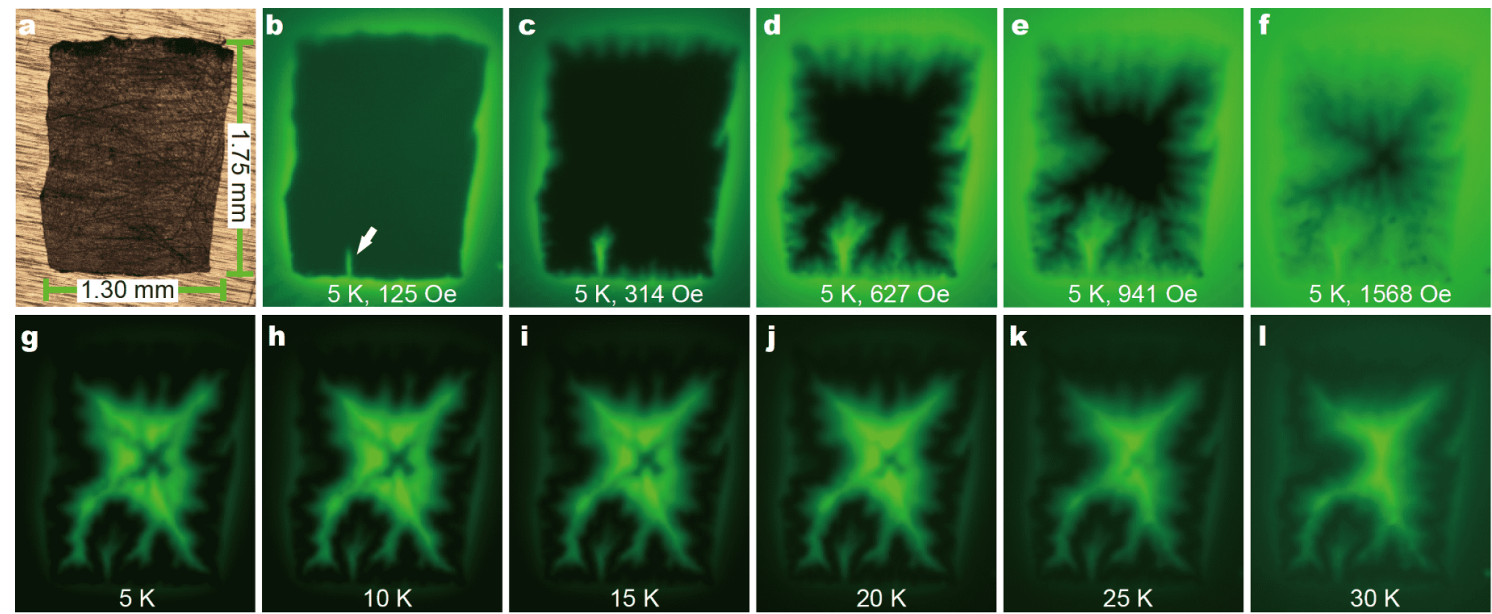

Figure 5 (a) Optical micrograph of the MO sample with a dimension of $1.75 \mathrm{~mm} \times 1.30 \mathrm{~mm} \times 0.025 \mathrm{~mm}$. (b-f) MO images showing flux penetration after zero-field-cooling the sample to $5 \mathrm{~K}$ and applying magnetic field. ( $\mathrm{g}-\mathrm{l})$ The remanent states obtained by decreasing the field from 1568 Oe to zero between 5 and $30 \mathrm{~K}$.

produced by polishing. The flux begins to penetrate gradually into the whole sample at 314 Oe. Fig. $5 \mathrm{c}-\mathrm{f}$ clearly show the flux entering from the edges into the center in a dendritic path. However, flux is still precluded from the center region even at the maximum attainable field, indicating a large circulating current inside the Ba122 bulk. The MO images of the sample in the remanent state obtained by decreasing the field from 1568 Oe to zero between 5 and $30 \mathrm{~K}$ are shown in Fig. $5 \mathrm{~g}-$ 1. A rooftop pattern of the magnetic flux was observed up to $30 \mathrm{~K}$, indicating a uniform global current flow throughout the whole sample. It is noteworthy that the pattern of the trapped flux changes little below $20 \mathrm{~K}$. Even at $25 \mathrm{~K}$, the magnetic flux still cannot penetrate the entire sample, indicating that tape-1.9-mm has pretty good performance at high temperatures.

The resistance transition curves of the sample tape-1.9$\mathrm{mm}$ in different magnetic fields were measured to evaluate the upper critical field $H_{\mathrm{c} 2}$, anisotropy $\gamma$ and the pinning potential $U_{0}$. Fig. 6 a shows the $R$ - $T$ curves of different magnetic fields in directions of $\mathrm{B} / /$ tape and $\mathrm{B} \perp$ tape, showing a transition broadening with increasing magnetic fields. The upper critical field $H_{\mathrm{c} 2}$ and the irreversibility field $H_{\text {irr }}$ are defined by $90 \%$ and $10 \%$ of the normal state resistivity, as shown in Fig. 6b. By using the Werthamer-Helfand-Hohenberg formula $H_{\mathrm{c} 2}(0)=$ $-0.693 T_{\mathrm{c}}\left(\mathrm{d} H_{\mathrm{c} 2} / \mathrm{d} T\right)$ [32], with $\mathrm{d} H_{\mathrm{c} 2} / \mathrm{d} T$ at $T=T_{\mathrm{c}}$, the $H_{\mathrm{c} 2}{ }^{\prime \prime}(0)$ and $H_{\mathrm{c} 2}{ }^{\perp}(0)$ are estimated to be 240 and $110 \mathrm{~T}$, which are very high values even compared with $(\mathrm{Ba}, \mathrm{K})$ $\mathrm{Fe}_{2} \mathrm{As}_{2}$ single crystals [33]. The anisotropy is defined as $\gamma$ $=H_{\mathrm{c} 2}{ }^{11} / H_{\mathrm{c} 2}{ }^{\perp}$ and varies from 2.0 to 3.6 at temperatures from 37.7 to $38.4 \mathrm{~K}$, as plotted in the inset. Actually, $\gamma$ of Ba122 decreases with decreasing temperature [11], which means that the anisotropy of the sample tape-1.9-mm will be less than 2 at temperatures below $37.7 \mathrm{~K}$. The results are quite similar to those in $(\mathrm{Ba}, \mathrm{K}) \mathrm{Fe}_{2} \mathrm{As}_{2}$ single crystals, suggesting that Ba122 has small anisotropy as its intrinsic property. Furthermore, since the resistance in the lowresistance region is induced by the creep of vortices [34], the temperature dependences of the resistivity can be described as the equation $\rho(T, B)=\rho_{0} \exp \left[-U_{0} / k_{\mathrm{B}} T\right]$, where $\rho_{0}$ is a parameter, $k_{\mathrm{B}}$ is the Boltzmann constant and $U_{0}$ is the flux-flow activation energy. By plotting $\ln \rho(T, B)$ as a function of $1 / T$, one can obtain $U_{0} / k_{\mathrm{B}}$ from the slop of the linear part, as shown in Fig. 6c. The values of the activation energy $U_{0} / k_{\mathrm{B}}$ range from $8500 \mathrm{~K}$ at $0.1 \mathrm{~T}$ to $3100 \mathrm{~K}$ at $9 \mathrm{~T}$ for the parallel fields and from $7500 \mathrm{~K}$ at $0.1 \mathrm{~T}$ to $2500 \mathrm{~K}$ at $9 \mathrm{~T}$ for the vertical fields, showing relatively weak field dependence compared with $\mathrm{MgB}_{2}$ [35]. For comparison, $U_{0}$ values for Sr122 [36], YBCO [37], Bi2212 [38] and $\mathrm{MgB}_{2}$ [35] are included in Fig. 6d. To summarize, these results indicate that our Ba122 tapes have very high intrinsic flux-pinning strength, high upper critical field $H_{\mathrm{c} 2}$, low anisotropy $\gamma$ and large activation energy $U_{0} / k_{\mathrm{B}}$, which are the fundamental reasons for the high performance in high magnetic fields.

The flux pinning mechanism in tape-1.9-mm was analyzed based on the Dew-Hughes model [39]. First of all, the isothermal hysteresis loops of the tape were measured and the magnetic critical current density $J_{\mathrm{c}}^{\mathrm{mag}}$ was calculated based on the Bean model with the equation $J_{\mathrm{c}}^{\mathrm{mag}}=20 \Delta M / w(1-w / 3 l)[40]$, where $\Delta M$ is the dif- 

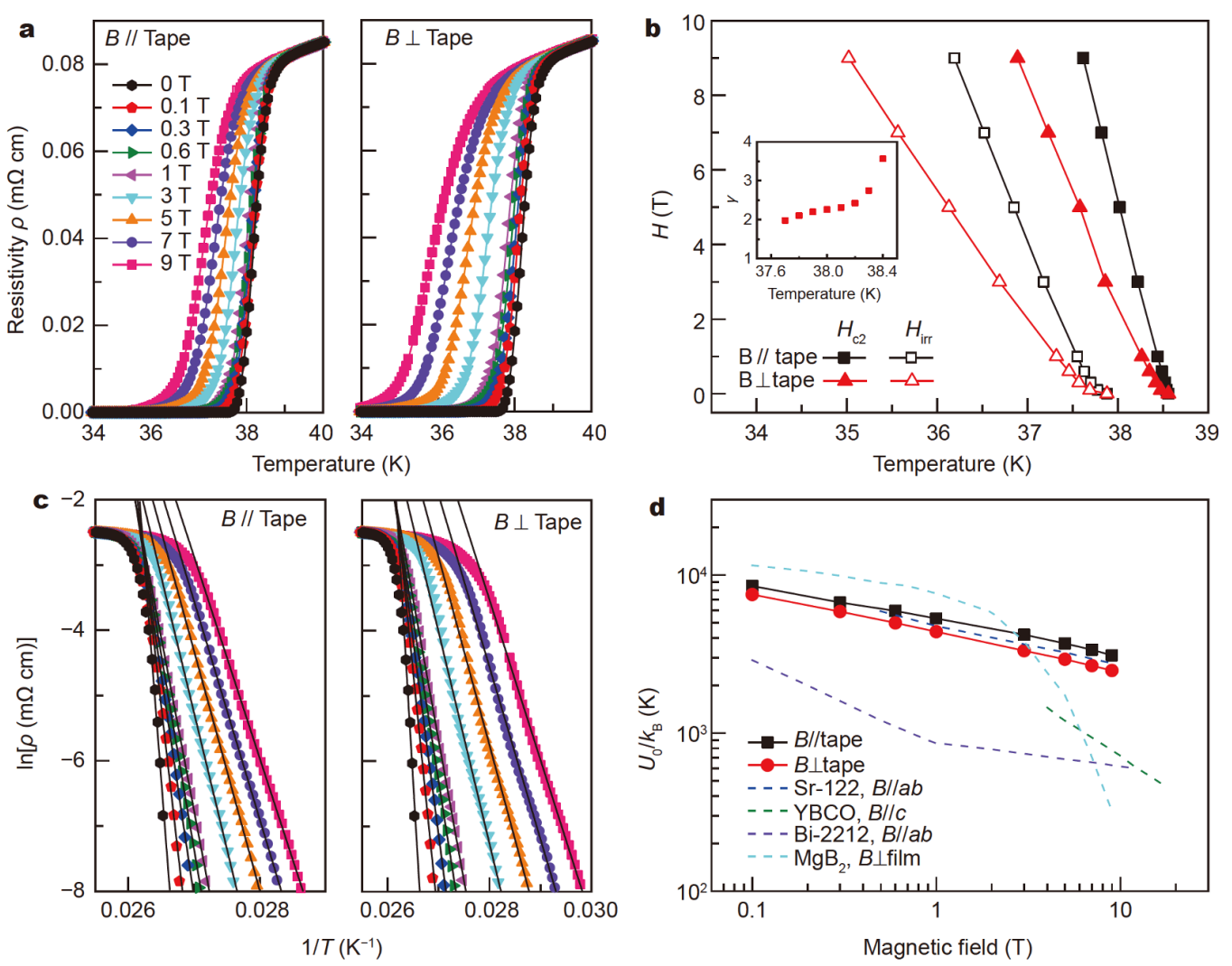

Figure 6 (a) The temperature-dependent resistivity of tape-1.9-mm in various magnetic fields for $B / /$ tape and $B \perp$ tape. (b) $H_{\mathrm{c} 2}$ and $H_{\text {irr }}$ for both field directions. The inset presents the anisotropy $\gamma=H_{\mathrm{c} 2}{ }^{\prime \prime} / H_{\mathrm{c} 2}{ }^{\perp}$ at different magnetic fields. (c) The Arrhenius plot to define the values of $U_{0} / k_{\mathrm{B}}$. (d) The pinning potential $U_{0} / k_{\mathrm{B}}$ as a function of magnetic field.

ference between the upper and lower branches of the hysteresis loops, $w$ and $l$ are the width and length of the sample $(l>w)$, respectively. The irreversibility field $H_{\text {irr }}$ is determined by the linear part of the Kramer plot: $K_{\mathrm{r}}(\mathrm{B})=$ $J_{\mathrm{c}}^{1 / 2} \mathrm{~B}^{1 / 4}$ at $K_{\mathrm{r}}=0$, as shown in the inset of Fig. 7. Fig. 7 presents the normalized pinning force density $f=F_{\mathrm{p}} /$ $F_{\mathrm{p}, \max }$ as a function of $h=H / H_{\text {irr }}$. The data points can be fitted by the equation $f=\mathrm{A} h^{p}(1-h)^{q}$ with $p=0.47, q=$ 1.87. The fitting curve peaks at $h_{0}=0.20$, indicating that the pinning mechanism is more close to the grain boundary pinning when compared with the hot pressed tape whose $h_{0}$ locates at $0.22[25,27,41]$. This is in accordance with the fact that the sample tape-1.9-mm has relatively small grains. Smaller grains correspond to increased grain boundary density and thus are favorable for enhancing the pinning force. As we put forward above, texture is critical to the transport performance of the Ba122 tapes. Therefore, one can conclude that these $c$ axis-textured small grains of $\sim 0.5 \mu \mathrm{m}$ in size in Fig. $4 \mathrm{a}$ are the desired ones and account for the high transport performance. On the other hand, for those tiny grains (smaller than $0.2 \mu \mathrm{m}$ in size) which have high-angle grain boundaries with adjacent grains, if they can be transformed to be $c$-axis textured to reduce the weak-linked grain boudaries, the transport performance of the Ba122 tape will definitely be further improved because these tiny grains can provide more grain boundaries as the flux pinning centers.

\section{DISCUSSION}

One of the major achievements of this work is that the transport $J_{\mathrm{c}}$ exceeding $10^{5} \mathrm{~A} \mathrm{~cm}^{-2}(4.2 \mathrm{~K}, 10 \mathrm{~T})$ was achieved in $\mathrm{Cu} / \mathrm{Ag}$ composite-sheathed Ba122 tapes, which is currently a record value for low-cost $\mathrm{Cu} / \mathrm{Ag}$ composite IBS tapes and wires. This value also exceeds a widely accepted threshold for practical applications, and more importantly was achieved through a scalable fabrication route. Besides, relevant characterizations have shown that these tapes have pure Ba122 phase with high density and good uniformity. In this case, because the extrinsic factors were eliminated as much as possible, we emphasized the significant role of grain texture in 


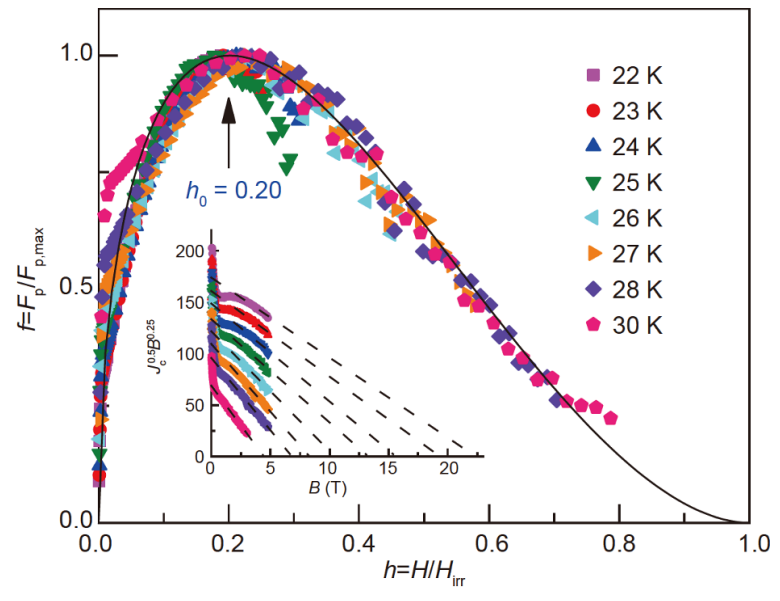

Figure 7 Normalized pinning force density $f=F_{\mathrm{p}} / F_{\mathrm{p}, \max }$ as a function of the reduced field $h=H / H_{\text {irr. }}$ The inset shows the Kramer plot to define the $H_{\text {irr }}$. The black solid line is the fitting curve using the equation $f=\mathrm{A} h^{p}(1-h)^{q}$, whose peak appears at $h=0.20$.

achieving high transport $J_{c}$. Another distinct feature of our tapes is the small grain size (mostly $\sim 0.5 \mu \mathrm{m}$ ). Based on the pinning mechanism analysis showing the grain boundaries as the main pinning centers, we put forward that small grains are beneficial to the transport performance of Ba122 tapes, provided that they are textured.

Despite the many favorable properties in the highest $-J_{c}$ tape-1.9-mm, several performance-hindering features were also found, suggesting that the $J_{c}$ value of HIP-sintered Ba122 tapes is still far from reaching its maximum. As revealed in both XRD and EBSD analyses, HIP-sintered Ba122 tapes have a relatively low degree of texture than the hot-pressed tapes with a record $J_{\mathrm{c}}$ value as high as $1.5 \times 10^{5} \mathrm{~A} \mathrm{~cm}^{-2}(4.2 \mathrm{~K}, 10 \mathrm{~T})$ [24]. This can be ascribed to the different ways in which high pressure was applied. For HIP-sintered Ba122 tapes, the high isostatic pressure provided by argon may not contribute much to further improve the grain texture. In order to enhance the texture, more attention should be paid to the optimization of rolling process since it is the main means of introducing pre-texture. Increasing the wire diameter before rolling in this work is a proven way to obtain higher pre-texture in Ba122 tapes. Reducing the final tape thickness may also produce similar benefits, but will face some difficulties in deformation uniformity.

\section{CONCLUSION}

In summary, $\mathrm{Cu} / \mathrm{Ag}$ composite-sheathed Ba122 ironpnicted tapes with practical-level transport $J_{\mathrm{c}}$ were prepared through a scalable and cost-effective fabrication route that includes ex situ PIT method, cold rolling process and HIP technique. By tuning the rolling-induced grain alignment, it is found that the transport $J_{c}$ of the tapes is closely and positively correlated to the degree of grain texture, proving that grain texture plays a critical role in improving $J_{\mathrm{c}}$ for iron-based superconducting wires and tapes. Detailed characterizations show that the highest $-J_{\mathrm{c}}$ sample tape-1.9-mm has pure Ba122 phase with high density and good uniformity, excellent grain connectivity, favorable performance at temperatures up to $25 \mathrm{~K}$, ultrahigh $H_{\mathrm{c} 2}$ up to $240 \mathrm{~T}$, low anisotropy and high pinning potential. Quantitative analysis by means of EBSD reveals that $c$-axis-textured small grains $(\sim 0.5 \mu \mathrm{m})$ account for the high transport $J_{c}$. On the other hand, several performance-hindering features were also found, such as relatively low degree of texture compared with hot-pressed tapes and randomly oriented tiny grains (less than $0.2 \mu \mathrm{m}$ ). Therefore, there is still room for the enhancement of the transport properties of $\mathrm{Cu} / \mathrm{Ag}$ composite-sheathed Ba122 tapes. This work proves the feasibility of large-scale low-cost preparation of highperformance IBS superconductors and will certainly promote the practical research of IBSs.

Received 29 December 2020; accepted 7 February 2021; published online 28 May 2021

1 Kamihara $\mathrm{Y}$, Watanabe $\mathrm{T}$, Hirano $\mathrm{M}$, et al. Iron-based layered superconductor $\mathrm{La}\left[\mathrm{O}_{1-x} \mathrm{~F}_{x}\right] \mathrm{FeAs}(x=0.05-0.12)$ with $T_{\mathrm{c}}=26 \mathrm{~K}$. $\mathrm{J}$ Am Chem Soc, 2008, 130: 3296-3297

2 Chen X, Dai P, Feng D, et al. Iron-based high transition temperature superconductors. Natl Sci Rev, 2014, 1: 371-395

3 Gurevich A. Challenges and opportunities for applications of unconventional superconductors. Annu Rev Condens Matter Phys, 2014, 5: 35-56

4 Hosono H, Yamamoto A, Hiramatsu H, et al. Recent advances in iron-based superconductors toward applications. Mater Today, 2018, 21: 278-302

5 Si Q, Yu R, Abrahams E. High-temperature superconductivity in iron pnictides and chalcogenides. Nat Rev Mater, 2016, 1: 16017

6 Ren ZA, Zhao ZX. Research and prospects of iron-based superconductors. Adv Mater, 2009, 21: 4584-4592

7 Sasmal K, Lv B, Lorenz B, et al. Superconducting Fe-based compounds $\left(\mathrm{A}_{1-x} \mathrm{Sr}_{x}\right) \mathrm{Fe}_{2} \mathrm{As}_{2}$ with $\mathrm{A}=\mathrm{K}$ and $\mathrm{Cs}$ with transition temperatures up to $37 \mathrm{~K}$. Phys Rev Lett, 2008, 101: 107007

8 Rotter M, Tegel M, Johrendt D. Superconductivity at $38 \mathrm{~K}$ in the iron arsenide $\left(\mathrm{Ba}_{1-x} \mathrm{~K}_{x}\right) \mathrm{Fe}_{2} \mathrm{As}_{2}$. Phys Rev Lett, 2008, 101: 107006

9 Tarantini C, Gurevich A, Jaroszynski J, et al. Significant enhancement of upper critical fields by doping and strain in ironbased superconductors. Phys Rev B, 2011, 84: 184522

10 Yamamoto A, Jaroszynski J, Tarantini C, et al. Small anisotropy, weak thermal fluctuations, and high field superconductivity in Codoped iron pnictide $\mathrm{Ba}\left(\mathrm{Fe}_{1-x} \mathrm{Co}_{x}\right)_{2} \mathrm{As}_{2}$. Appl Phys Lett, 2009, 94: 062511

11 Yuan HQ, Singleton J, Balakirev FF, et al. Nearly isotropic superconductivity in (Ba,K) $\mathrm{Fe}_{2} \mathrm{As}_{2}$. Nature, 2009, 457: 565-568 
12 Ma Y. Progress in wire fabrication of iron-based superconductors. Supercond Sci Technol, 2012, 25: 113001

13 Pallecchi I, Eisterer M, Malagoli A, et al. Application potential of Fe-based superconductors. Supercond Sci Technol, 2015, 28: 114005

14 Malagoli A, Wiesenmayer E, Marchner S, et al. Role of heat and mechanical treatments in the fabrication of superconducting $\mathrm{Ba}_{0.6^{-}}$ $\mathrm{K}_{0.4} \mathrm{Fe}_{2} \mathrm{As}_{2}$ ex situ powder-in-tube tapes. Supercond Sci Technol, 2015, 28: 095015

15 Bonura M, Huang H, Yao C, et al. Current transport, magnetic and elemental properties of densified Ag-sheathed $\mathrm{Ba}_{1-x} \mathrm{~K}_{x} \mathrm{Fe}_{2} \mathrm{As}_{2}$ tapes. Supercond Sci Technol, 2020, 33: 095008

16 Durrell JH, Eom CB, Gurevich A, et al. The behavior of grain boundaries in the Fe-based superconductors. Rep Prog Phys, 2011, 74: 124511

17 Katase T, Ishimaru Y, Tsukamoto A, et al. Advantageous grain boundaries in iron pnictide superconductors. Nat Commun, 2011, 2: 409

18 Hiramatsu H, Katase T, Ishimaru Y, et al. Microstructure and transport properties of [001]-tilt bicrystal grain boundaries in iron pnictide superconductor, cobalt-doped $\mathrm{BaFe}_{2} \mathrm{As}_{2}$. Mater Sci Eng-B, 2012, 177: 515-519

19 Shabbir B, Huang H, Yao C, et al. Evidence for superior current carrying capability of iron pnictide tapes under hydrostatic pressure. Phys Rev Mater, 2017, 1: 044805

20 Hecher J, Baumgartner T, Weiss JD, et al. Small grains: A key to high-field applications of granular Ba-122 superconductors? Supercond Sci Technol, 2016, 29: 025004

21 Wang L, Qi Y, Zhang X, et al. Textured $\mathrm{Sr}_{1-x} \mathrm{~K}_{x} \mathrm{Fe}_{2} \mathrm{As}_{2}$ superconducting tapes with high critical current density. Physica CSupercond, 2011, 471: 1689-1691

22 Gao Z, Ma Y, Yao C, et al. High critical current density and low anisotropy in textured $\mathrm{Sr}_{1-x} \mathrm{~K}_{x} \mathrm{Fe}_{2} \mathrm{As}_{2}$ tapes for high field applications. Sci Rep, 2012, 2: 998

23 Zhang $\mathrm{X}$, Yao C, Lin $\mathrm{H}$, et al. Realization of practical level current densities in $\mathrm{Sr}_{0.6} \mathrm{~K}_{0.4} \mathrm{Fe}_{2} \mathrm{As}_{2}$ tape conductors for high-field applications. Appl Phys Lett, 2014, 104: 202601

24 Gao Z, Togano K, Zhang Y, et al. High transport $J_{c}$ in stainless steel/Ag-Sn double sheathed Ba122 tapes. Supercond Sci Technol, 2017, 30: 095012

25 Huang H, Yao C, Dong C, et al. High transport current superconductivity in powder-in-tube $\mathrm{Ba}_{0.6} \mathrm{~K}_{0.4} \mathrm{Fe}_{2} \mathrm{As}_{2}$ tapes at $27 \mathrm{~T}$. Supercond Sci Technol, 2018, 31: 015017

26 Weiss JD, Tarantini C, Jiang J, et al. High intergrain critical current density in fine-grain $\left(\mathrm{Ba}_{0.6} \mathrm{~K}_{0.4}\right) \mathrm{Fe}_{2} \mathrm{As}_{2}$ wires and bulks. Nat Mater, 2012, 11: 682-685

27 Liu S, Lin K, Yao C, et al. Transport current density at temperatures up to $25 \mathrm{~K}$ of $\mathrm{Cu} / \mathrm{Ag}$ composite sheathed 122-type tapes and wires. Supercond Sci Technol, 2017, 30: 115007

28 Pyon S, Suwa T, Tamegai T, et al. Improvements of fabrication processes and enhancement of critical current densities in $(\mathrm{Ba}, \mathrm{K})$ $\mathrm{Fe}_{2} \mathrm{As}_{2}$ HIP wires and tapes. Supercond Sci Technol, 2018, 31: 055016

29 Li L, Zhang X, Yao C, et al. Enhancement of the critical current density in $\mathrm{Cu} / \mathrm{Ag}$ composite sheathed $(\mathrm{Ba}, \mathrm{K}) \mathrm{Fe}_{2} \mathrm{As}_{2}$ tapes by preannealing process. Mater Res Express, 2019, 6: 096003

30 Liu S, Cheng Z, Yao C, et al. High critical current density in $\mathrm{Cu} / \mathrm{Ag}$ composited sheathed $\mathrm{Ba}_{0.6} \mathrm{~K}_{0.4} \mathrm{Fe}_{2} \mathrm{As}_{2}$ tapes prepared via hot isostatic pressing. Supercond Sci Technol, 2019, 32: 044007

31 Lotgering FK. Topotactical reactions with ferrimagnetic oxides having hexagonal crystal structures-I. J Inorg Nucl Chem, 1959, 9: $113-123$

32 Werthamer NR, Helfand E, Hohenberg PC. Temperature and purity dependence of the superconducting critical field, $\mathrm{H}_{\mathrm{c} 2}$. III. Electron spin and spin-orbit effects. Phys Rev, 1966, 147: 295-302

33 Wang XL, Ghorbani SR, Lee SI, et al. Very strong intrinsic flux pinning and vortex avalanches in $(\mathrm{Ba}, \mathrm{K}) \mathrm{Fe}_{2} \mathrm{As}_{2}$ superconducting single crystals. Phys Rev B, 2010, 82: 024525

34 Palstra TT, Batlogg B, van Dover RB , et al. Dissipative flux motion in high-temperature superconductors. Phys Rev B, 1990, 41: 66216632

35 Sidorenko A, Zdravkov V, Ryazanov V, et al. Thermally assisted flux flow in $\mathrm{MgB}_{2}$ : Strong magnetic field dependence of the activation energy. Philos Mag, 2005, 85: 1783-1790

36 Lin $\mathrm{H}$, Dong $\mathrm{C}$, Pan $\mathrm{X}$, et al. Strong flux pinning and anomalous anisotropy of $\mathrm{Sr}_{0.6} \mathrm{~K}_{0.4} \mathrm{Fe}_{2} \mathrm{As}_{2}$ superconducting tapes. Supercond Sci Technol, 2020, 33: 125001

37 López D, Krusin-Elbaum L, Safar H, et al. Pinned vortex liquid above the critical point of the first-order melting transition: A consequence of pointlike disorder. Phys Rev Lett, 1998, 80: 10701073

38 Palstra TTM, Batlogg B, Schneemeyer LF, et al. Thermally Activated Dissipation in $\mathrm{Bi}_{2.2} \mathrm{Sr}_{2} \mathrm{Ca}_{0.8} \mathrm{Cu}_{2} \mathrm{O}_{8+\delta}$. Phys Rev Lett, 1988, 61: 1662-1665

39 Dew-Hughes D. Flux pinning mechanisms in type II superconductors. Philos Mag, 1974, 30: 293-305

40 Bean CP. Magnetization of high-field superconductors. Rev Mod Phys, 1964, 36: 31-39

41 Dong $\mathrm{C}$, Lin $\mathrm{H}$, Huang $\mathrm{H}$, et al. Vortex pinning and dynamics in high performance $\mathrm{Sr}_{0.6} \mathrm{~K}_{0.4} \mathrm{Fe}_{2} \mathrm{As}_{2}$ superconductor. J Appl Phys, 2016, 119: 143906

Acknowledgements This work was supported by the National Key R\&D Program of China (2018YFA0704200 and 2017YFE0129500), the National Natural Science Foundation of China (51861135311, U1832213 and 51721005), the Strategic Priority Research Program of Chinese Academy of Sciences (XDB25000000), and the Key Research Program of Frontier Sciences of Chinese Academy of Sciences (QYZDJ-SSWJSC026).

Author contributions Liu S designed the experimental plan, and performed sample preparation and most of the characterizations. Yao C and Dong $\mathrm{C}$ provided significant guidance on data analysis and coauthored this article. Huang $\mathrm{H}$ and Guo $\mathrm{W}$ measured the critical currents of the Ba122 tapes at $4.2 \mathrm{~K}$ under external magnetic fields. Cheng $\mathrm{Z}$ and Zhu Y contributed to the preparation of Ba122 precursor. Awaji S provided the high-field critical current test system and gave useful advice on the critical current measurements. Ma Y directed the project and also provided significant guidance on data analysis and article writing.

Conflict of interest The authors declare that they have no conflict of interest. 


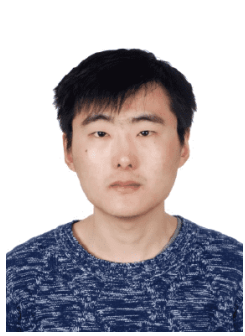

Shifa Liu received his BSc degree from the School of Materials Science and Engineering of Tsinghua University and is currently a $\mathrm{PhD}$ candidate at the Institute of Electrical Engineering, Chinese Academy of Sciences and University of Chinese Academy of Sciences under the supervision of Prof. Yanwei Ma. Liu's research work focuses on the practical research of ironbased superconductors, particularly through the hot isostatic pressing technique.

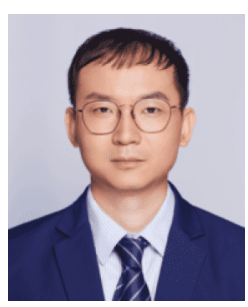

Chao Yao is an associate professor at the Institute of Electrical Engineering, Chinese Academy of Sciences. He received his $\mathrm{PhD}$ from the University of Chinese Academy of Sciences in 2014 and B.Eng. from Beijing University of Posts and Telecommunications in 2008. $\mathrm{He}$ is a member of Youth Innovation Promotion Association, Chinese Academy of Sciences. His research focuses on the fabrication of highperformance iron-based superconducting wires based on powder-in-tube method.

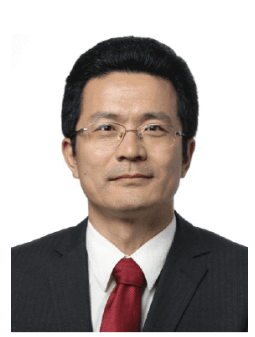

Yanwei $\mathrm{Ma}$ is a professor at the Institute of Electrical Engineering, Chinese Academy of Sciences. He received his $\mathrm{PhD}$ degree from Tsinghua University in 1996. In 1998-2004, he worked at the Institute for Materials Research, Tohoku University (Sendai, Japan), National Institute for Materials Science (Tsukuba, Japan) and Universite de Rennes 1 (France), respectively. His group is specialized in the development of superconducting wires and nanomaterials for energy storage, and fabrication of graphene-based supercapacitors. He currently serves as international board member for many prestigious journals, such as Superconductor Science and Technology, Physica C: Superconductivity and its Applications, etc.

\section{$\mathrm{Ba}_{1-x} \mathrm{~K}_{x} \mathrm{Fe}_{2} \mathrm{As}_{2}$ 铁基超导带材的织构调控与性能} 研究

刘世法 ${ }^{1,2}$, 姚超 ${ }^{1,2^{*}}$, 黄河 ${ }^{1}$, 董持衡 ${ }^{1,2}$, 郭文文 ${ }^{1,2}$, 成者 ${ }^{1,2}$, 朱炎昌 ${ }^{1}$, Satoshi Awaji ${ }^{3}$, 马衍伟 ${ }^{1,2}$

摘要 高性能超导材料是高场磁体开发的基础. 铁基超导体具有很 高的上临界场和较低的各向异性，在高场应用领域具有广阔的前 景, 如加速器磁体、高场核磁共振谱仪、医用磁共振成像系统和 可控核聚变装置. 本文通过一种易于规模扩展的工艺路线制备了 铜银复合包套 $\mathrm{Ba}_{1-x} \mathrm{~K}_{x} \mathrm{Fe}_{2} \mathrm{As}_{2}$ (Ba122)超导带材, 其传输临界电流密 度在 $4.2 \mathrm{~K}$ 和 $10 \mathrm{~T}$ 外磁场下为 $1.1 \times 10^{5} \mathrm{~A} \mathrm{~cm}^{-2}$, 达到了 $10^{5} \mathrm{~A} \mathrm{~cm}^{-2}$ 的实 用化水平. 该制备路线结合了平辊轧制织构化工艺和热等静压致 密化工艺. 通过改变轧制工艺参数, 可以调控多晶的织构度. 研究 表明, 在轧制过程中施加较大的变形量可以提高Ba122超导带材的 $c$ 轴织构度, 从而提高其传输性能. 对高性能带材的微观结构表征表 明, 其Ba122相元素分布均匀, 晶粒细小且连接性良好, 有助于提高 晶界钉扎作用. 磁光成像研究表明, 即使在 $25 \mathrm{~K}$, 样品中仍然存在较 大的磁化电流. 本文采用的易于规模化扩展且低成本的制备路线 在Ba122超导带材中获得了很高的传输性能, 表明铁基超导材料在 未来高场应用中是潜在的有力竞争者. 